\title{
Efficacy and Safety of Mizoribine by One Single Dose Administration for Patients with Rheumatoid Arthritis
}

\author{
Kunihiro Ichinose ${ }^{1}$, Tomoki Origuchi ${ }^{2}$, Shin-ya Kawashiri ${ }^{1}$, Naoki Iwamoto ${ }^{1}$, \\ Keita Fujikawa ${ }^{1}$, Toshiyuki Aramaki ${ }^{1}$, Makoto Kamachi ${ }^{1}$, Kazuhiko Arima ${ }^{1}$, Mami Tamai ${ }^{1}$, \\ Hideki Nakamura ${ }^{1}$, Hiroaki Ida ${ }^{1}$, Atsushi Kawakami ${ }^{1}$, Toshiaki Tsukada ${ }^{3}$, Yukitaka Ueki ${ }^{4}$ \\ and Katsumi Eguchi ${ }^{1}$
}

\begin{abstract}
Objective Mizoribine (MZR) is an immunosuppressant that inhibits nucleic acid metabolism and is a relatively safe disease-modifying anti-rheumatic drug (DMARD). We evaluated the efficacy and safety of one single dose per day for patients with rheumatoid arthritis (RA).

Patients and Methods In this study 32 patients with RA received MZR therapy. We evaluated the average dose of MZR and prednisolone, response to treatment and peak plasma level of MZR.

Results The average dose of MZR was $146.1 \pm 31.2$ (range: 50-200) $\mathrm{mg} /$ day. The average dose of prednisolone was $4.63 \pm 3.59$ (range: $0-14$ ) $\mathrm{mg} /$ day. The average plasma level of MZR, measured after 3 hours, was $2.20 \pm 0.49 \mu \mathrm{g} / \mathrm{mL}$ in the responder group and $1.59 \pm 0.82 \mu \mathrm{g} / \mathrm{mL}$ in the non-responder group $(\mathrm{p}=0.020)$. The treatment with MZR for 24 weeks was completed by $71.9 \%$ of patients and the proportion of patients who achieved a good and moderate response rate according to the European League Against Rheumatism (EULAR) criteria was $56.3 \%$ at 24 weeks. The plasma level of MZR which was greater than or equal to $2.12 \mu \mathrm{g} /$ $\mathrm{mL}$ was significantly correlated with the clinical response $(\mathrm{p}<0.01)$. Only one of thirty-two cases discontinued the treatment, because of skin eruption.

Conclusion This study included patients that could not be treated with other DMARDs and/or biologic agents because of age, interstitial pneumonia and other complications. We show that MZR may be a useful and relatively safe therapy for patients in this group.
\end{abstract}

Key words: rheumatoid arthritis (RA), mizoribine (MZR), plasma level

(Inter Med 49: 2211-2218, 2010)

(DOI: 10.2169/internalmedicine.49.3810)

\section{Introduction}

Mizoribine (MZR) is a novel immunosuppressive agent which was isolated from the culture media of Eupenicillum brefeldianum M-2166 in 1974 in Japan (1). This agent inhibits inosine monophosphate dehydrogenasase (IMPDH), a rate-limiting enzyme in the de novo pathway of nucleic acid synthesis $(2,3)$. The immunosuppressive effect of MZR has been suggested to be due to the inhibition of $\mathrm{T}$ and $\mathrm{B}$ cell proliferation (4). The clinical efficacy of MZR was first approved in renal transplant recipients $(4,5)$. Since then MZR has been thought to be safe and well tolerated compared with other immunosuppressants, and recent studies have demonstrated the utility in the treatment of rheumatoid arthritis (RA) (6), systemic lupus erythematosus (SLE) (7), nephrotic syndrome (8), and immunoglobulin A (IgA) nephropathy (9). Although, MZR has been considered a secondline disease-modifying anti-rheumatic drug (DMARD), it is often used in combination with methotrexate (MTX) or bio-

\footnotetext{
${ }^{1}$ Department of Rheumatology, Unit of Translational Medicine, Graduate School of Biomedical Sciences, Nagasaki University, Nagasaki, ${ }^{2}$ Department of Rehabilitation, Unit of Translational Medicine, Graduate School of Biomedical Sciences, Nagasaki University, Nagasaki, ${ }^{3}$ Isahaya Health Insurance General Hospital, Isahaya and ${ }^{4}$ Sasebo Chuo Hospital, Sasebo

Received for publication April 14, 2010; Accepted for publication June 16, 2010

Correspondence to Dr. Kunihiro Ichinose, kunizo1234abcd@yahoo.co.jp
} 
logical RA treatments in Japan $(10,11)$.

MZR can easily transfer from plasma to synovial fluid in RA patients. A previous report showed the ratio of drug concentration in plasma and synovial fluid of 15 RA patients was 1:0.84 for MZR, 1:0.56 for gold sodium thiomalate, 1:0.49 for methotrexate, 1:0.50 for Dpenicillamine and 1:0.31 for bucillamine (12).

In addition to increased tissue distribution, recent studies suggest that the peak plasma level of MZR is important for the clinical efficacy of this drug $(7,13)$. Human mixedlymphocyte reaction was shown to be inhibited with MZR at rates ranging from 36.4 to $62.2 \%$ in the range of $0.1-5$ $\mu \mathrm{g} / \mathrm{mL}$, and with $50 \%$ inhibition at about $1.0 \mu \mathrm{g} / \mathrm{mL}$ (14). According to an ex vivo study, regular use $(150 \mathrm{mg} /$ day, 3 divided doses) of MZR did not reach a high enough peak plasma level $(<0.8 \mu \mathrm{g} / \mathrm{mL})$ (15). Moreover, changing MZR administration from three divided doses to one single dose induced remission of relapsed membraneous nephropathy (16). This pilot study was aimed to investigate the efficacy and safety of MZR in 32 RA patients by changing the administration from three divided doses to one single dose in order to gain a higher plasma level of the drug.

\section{Abbreviations}

MZR: mizoribine, RA: rheumatoid arthritis, MTX: methotrexate

\section{Patients and Methods}

\section{Patients}

This pilot study was conducted from 2005 to 2007 at 3 institutions in Nagasaki, Japan. All patients who entered into this study had met the following inclusion criteria at the time of their enrollment in the preceding study: 1) a diagnosis of RA according to the American College of Rheumatology (ACR) criteria (revised in 1987), 2) a disease duration of at least 6 months, and 3) active disease (which was defined by at least six tender joints, at least three swollen joints and an erythrocyte sedimentation rate $>30 \mathrm{~mm} / \mathrm{h}$ or a C-reactive protein level $>1.0 \mathrm{mg} / \mathrm{dL}$ ). Those patients who had, severe drug hypersensitivity, bone marrow suppression, severe liver dysfunction, severe infection, pregnancy or malignancy were excluded from this study.

\section{Method}

Prior to the present study, the Institutional Review Board at each participating institution approved the study protocol. All of the patients involved gave written informed consent.

MZR was initiated at a dose of $50-200 \mathrm{mg}$ orally once a day after the morning meal. Dose regulation was possible depending on the patient's symptoms or adverse drug reactions at the discretion of the investigator. The duration of treatment in the study was scheduled to be 24 weeks. The nonsteroidal anti-inflammatory drugs (NSAIDs) and steroids that were used prior to the study could be continued. Dose reduction was allowed for NSAIDs and steroids during the study period. Concomitant use of other DMARDs (including gold salts, D-penicillamine, bucillamine, salazosulfapyridine, lobenzarit, actarit, cyclosporine, methotrexate and tacrolimus) was permitted when necessary. In patients who used other DMARDs together, we performed the evaluation after adding MZR. Administration of biologic agents that could influence the curative effect was prohibited.

\section{Abbreviations}

DMARDs: disease modifying anti-rheumatic drugs, NSAIDs: nonsteroidal anti-inflammatory drugs

\section{Evaluation}

Disease activity was assessed by the swollen and tender joint counts, the C-reactive protein level, and the Disease Activity Score in 28 joints (DAS28) following ACR guidelines, at the beginning of the study and at 4-week intervals thereafter to the end of the study ( 24 weeks). At the same intervals, global assessment of disease activity by the investigator, global assessment of disease activity by the patient, and assessment of joint tenderness by the patient were also recorded. In every examination, clinical improvement was assessed according to the European League Against Rheumatism (EULAR) response criteria. The responder group includes those patients who experienced a greater than or equal to moderate response after 24 weeks MZR treatment. The non-responder group includes the remaining patients. The peak plasma level of MZR was measured 3 hours after administration every 4 weeks of treatment, and we examined the association between EULAR response criteria and the blood concentration. All adverse events were examined and those for which a casual relationship to MZR could not be ruled out by the investigator were classified as adverse drug reactions.

\section{Statistical analysis}

The sample size of this pilot study was based on practical rather than biometric considerations. All values were expressed as mean +/- SEM. Kruskal-Wallis test with post-hoc comparisons using Scheffe's test and Fisher's exact test were employed for inter-group comparisons of multiple variables. Statistical analyses were performed by StatView software (Abacus Concepts, Berkeley, CA). A level of $\mathrm{p}<0.05$ was considered statistically significant.

\section{Results}

\section{Patient characteristics}

A total of 32 patients were enrolled in the present study. Treatment with MZR for 24 weeks was completed by $71.9 \%$ of the subjects (23/32 patients), while MZR was discontinued in $28.1 \%$ of them (9/32 patients) (Fig. 1). The mean age ( \pm standard deviation) of the patients was $64.5 \pm 10.7$ years (range: $32-85$ years) at enrollment in this study, and $68.7 \%$ (22/32 patients) had stage III or IV (evaluated by Steinbrocker's radiological stage) (17) RA at that time. The mean 
dosage of prednisolone was $4.63 \pm 3.59 \mathrm{mg} /$ day (range: $0-14$ $\mathrm{mg} /$ day). The mean dosage of MZR was $146.1 \pm 31.2 \mathrm{mg} /$ day (range: $50-200 \mathrm{mg} / \mathrm{day}$ ). DMARDs were used in 34.4\% (11/ 32) of patients and the mean number of DMARDs was $0.53 \pm 0.84$ (range: $0-3$ ). The numbers of patients who used DMARDs were as follows: salazosulfapyridine: 3 patients, methotrexate: 6 patients, bucillamine: 1 patient, gold salts: 2 patients. $59.4 \%$ had some complications, including interstitial pneumonia (25\%, 8/32 patients), osteoporosis (15.6\%, 5/ 32 patients), renal dysfunction $(9.3 \%, 3 / 32$ patients) and other pulmonary diseases $(9.3 \%, 3 / 32$ patients). The mean DAS28-CRP value (the 28 joint count Disease Activity Score using $C$ reactive protein) was $4.82 \pm 1.14$, the number

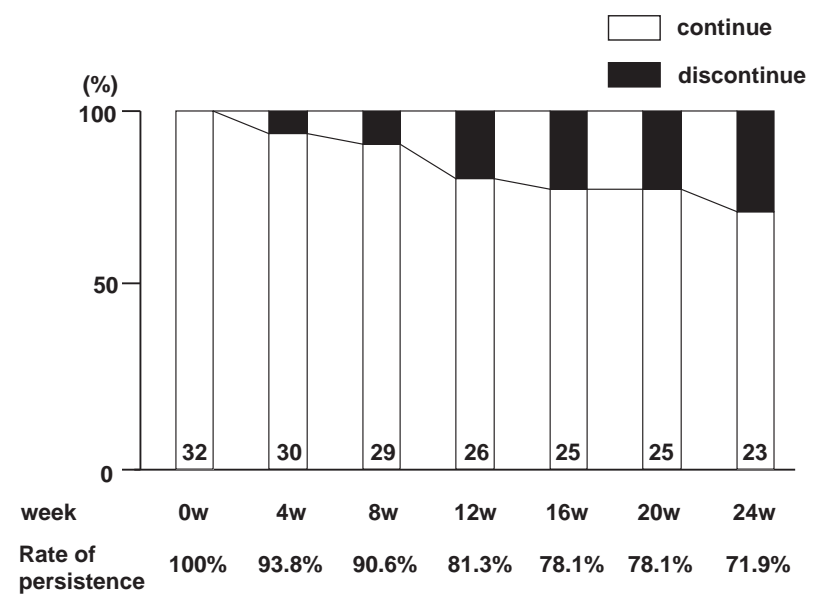

Figure 1. Rate of persistence after 24 weeks of mizoribine (MZR) treatment. The number of patients at each time point is shown in the bar. of tender joints was $9.07 \pm 7.42$, the number of swollen joints was $4.16 \pm 4.61$ and the visual analog scale (VAS) was $49.5 \pm$ $23.3 \mathrm{~mm}$ each, before the treatment. The mean C-reactive protein (CRP) level was $3.23 \pm 3.25 \mathrm{mg} / \mathrm{dL}$ (Table 1 ).

\section{Abbreviations}

CRP: C-reactive protein, DAS28-CRP: the 28 joint count Disease Activity Score using $\mathrm{C}$ reactive protein

\section{Safety}

All 32 patients were included in the safety analyses. The reasons for discontinuation of treatment were adverse events in $3.1 \%$ (1/32 patients), lack of improvement/worsening of RA in $21.8 \%$ (7/32 patients) and economic reason in $3.1 \%$ (1/32 patients) (Table 2). During the study period, no deaths occurred. No serious adverse events occurred. Only one adverse event occurred, which was skin eruption.

\section{Efficacy}

The median DAS28-CRP value showed a significant decrease after 16 weeks compared with that at baseline, and the effect continued to 24 weeks $(\mathrm{p}<0.05)$ (Fig. 2A). The median DAS-CRP values in the responder group showed a significant decrease compared with those in the nonresponder group throughout the treatment $(\mathrm{p}<0.05)$ (Fig. 2B). The median tender joint counts (TJC), swollen joint counts (SJC) and CRP were also significantly decreased over the 24 weeks of treatment (Fig. 3). Good and moderate response rates according to EULAR criteria were $31.2 \%$ (at 4 weeks), 53.1\% (at 12 weeks), and $56.3 \%$ (at 24 weeks) (Fig. 4). Comparing the reactivity of MZR, we found the plasma level of MZR in the responder group was higher than that in the non-responder group $(2.20 \pm 0.49 \mu \mathrm{g} /$

Table 1. Characteristics of the Patients at Enrollment in the Study

\begin{tabular}{l|c}
\hline Sex (male:female) & $6: 26$ \\
Age (years, mean \pm SD) & $64.5 \pm 10.7(32-85)$ \\
Duration of RA (years, mean \pm SD) & $14.0 \pm 10.9(2-40)$ \\
Steinbrocker stage (number) & I...0, II...10, III...9, IV ...13 \\
Dose of predonisolone (mg/day, mean \pm SD) & $4.63 \pm 3.59(0-14)$ \\
Dose of mizoribine (mg/day, mean \pm SD) & $146.1 \pm 31.2(50-200)$ \\
Tender joint counts ( mean \pm SD) & $9.07 \pm 7.42$ \\
Swollen joint counts ( mean \pm SD) & $4.16 \pm 4.61$ \\
C-reactive protein level (mg/dL, mean \pm SD) & $3.23 \pm 3.25$ \\
VAS (mm, mean \pm SD) & $49.5 \pm 23.3$ \\
DAS28-CRP ( mean \pm SD) & $4.82 \pm 1.14$ \\
\hline
\end{tabular}

VAS: visual analogue scale, DAS28-CRP: the 28 joint count Disease Activity Score using $C$ reactive protein

Table 2. The Reason for Discontinuation (of Patients 32 Analyzed)

\begin{tabular}{c|l}
\hline Reason of discontinuation & \multicolumn{1}{c}{ No. of patients (\%) } \\
\hline Lack of improvement/worsening of RA & $7 / 32(21.8 \%)$ \\
Adverse event & $1 / 32(3.1 \%):$ skin eruption \\
Economic reasons & $1 / 32(3.1 \%):$ It was not comparatively effective \\
\hline
\end{tabular}


A

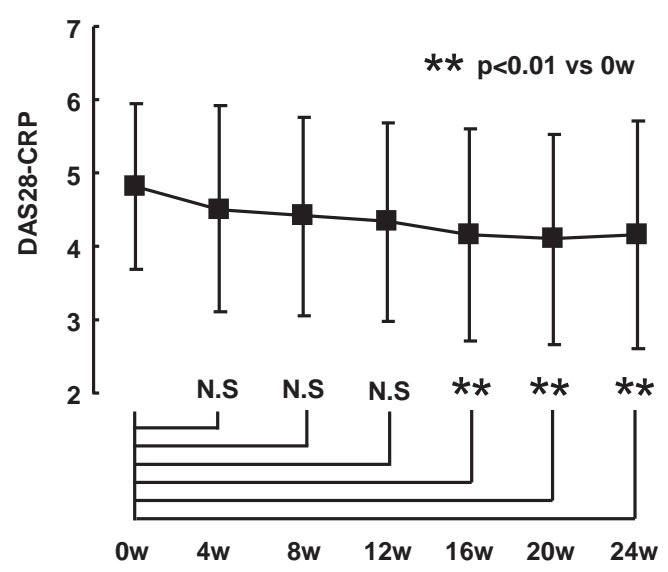

B

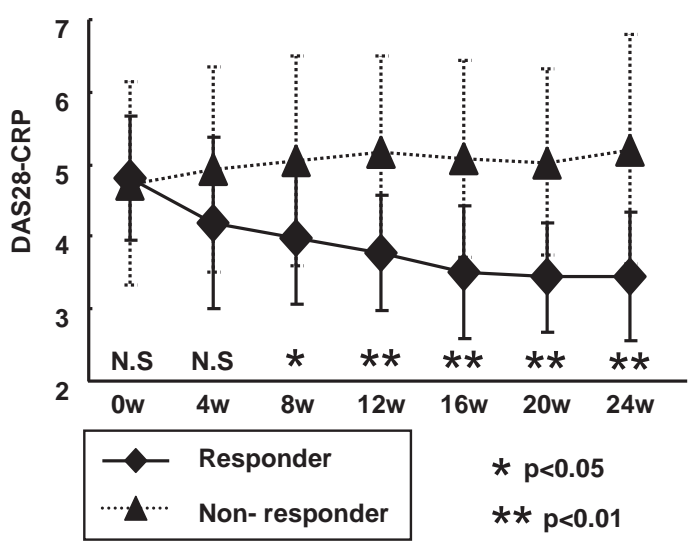

Figure 2. The efficacy value of the 28 joint count Disease Activity Score using $\mathrm{C}$ reactive protein (DAS28-CRP) for 24 weeks. (A) Total (B) Responders and non-responders. The responder group includes those patients who experienced a greater than or equal to moderate response after 24 weeks of mizoribine (MZR) treatment. The non-responder group includes the remaining patients. N.S: not significant, ${ }^{*} \mathbf{p}<0.05,{ }^{*}{ }^{*} \mathbf{p}<0.01,0$ week vs each week in $\mathrm{A}$, responder vs non-responder in B.

$\mathrm{mL}$ vs. $1.59 \pm 0.82 \mu \mathrm{g} / \mathrm{mL}, \mathrm{p}=0.020$ ) (Table 3). Next, we evaluated whether the clinical response correlated with the plasma level of MZR. Table 4 shows the analysis of clinical response and the plasma level of MZR. A plasma level of MZR greater than or equal to $2.12 \mu \mathrm{g} / \mathrm{mL}$ showed high positive and negative predictive values. Moreover, this plasma level of MZR showed high sensitivity and high specificity. We demonstrated that the plasma level of MZR which was greater than or equal to $2.12 \mu \mathrm{g} / \mathrm{mL}$ significantly correlated with clinical response $(\mathrm{p}<0.01$; Fig. 5$)$. No other characteristics of patients at baseline were significantly different between the responder group and the non-responder group.

\section{Discussion}

Mizoribine (MZR) is a novel immunosuppressive agent that is approved in Japan, South Korea and China for prevention of rejection in renal transplantation (5). Subsequently, MZR has been approved in Japan for the treatment of RA (6), SLE (7), nephrotic syndrome (8) and immunoglobulin A (IgA) nephropathy (9). This agent inhibits the proliferation of lymphocytes via inhibition of de novo purine biosynthesis selectively, and therefore it has been thought to inhibit both humoral and cellular immunity (2).

MZR is usually used at a daily dose of $150 \mathrm{mg}$ in three divided doses and has been thought to be safe and be well tolerated. However, its substantial clinical use remains relatively rare in comparison with other DMARDs and immunosuppressants because of its mild effect. Nakamura et al (18) and Nishimura (19) reported that RA patients who had not responded to a daily dose of $150 \mathrm{mg}$ in three divided doses were then found to show a good response to one single dose of $150 \mathrm{mg}$ MZR and showed obvious clinical response (greater than or equal to moderate response: 61.5\%) at 24 weeks. Recent studies suggest that the peak plasma level of MZR is important for the clinical efficacy of this drug $(7,13)$. In patients with lupus nephritis, the peak plasma level of MZR was correlated with the clinical response (20). MZR pulse therapy was also found to be effective in RA patients who showed only a partial response to MTX (21-23). In our report we found that MZR was effective and safe by a single dose administration for RA patients. We demonstrated that treatment with MZR for 24 weeks was completed by $71.9 \%$ of patients, of whom $56.3 \%$ achieved good or moderate response according to EULAR criteria at 24 weeks. Although patients of old age, with advanced-stage RA or with some complications were included in our study, the clinical efficacy and safety were similar to previous reports that administered a single dose of $\operatorname{MZR}(19,24,25)$.

Because pharmacokinetics in nine RA patients with normal renal function indicated that the MZR plasma level peaked at 2.4 hours after oral administration (26), we measured the peak plasma level 3 hours after administration. Additionally, with oral administration of $2.5-4.0 \mathrm{mg} / \mathrm{kg}$ per daily doses, the peak plasma levels of MZR usually showed less than $2.0 \mu \mathrm{g} / \mathrm{mL}$ in three divided doses. Therefore, our patients were administered a single dose of MZR at a dose of 50-200 mg orally once a day after the morning meal in order to increase the peak plasma level of MZR. No particular adverse events of MZR have been observed even at $6 \mu \mathrm{g} /$ $\mathrm{mL}$ or above (27), yet there is no reliable data concerning the upper limit of the peak plasma level of MZR. Our result clearly shows that most of the patients who achieved a good response required at least $2.12 \mu \mathrm{g} / \mathrm{mL}$ peak plasma level of MZR. A similar plasma level of MZR has been reported as an effective dose with lupus nephritis patients $(20,28)$. 


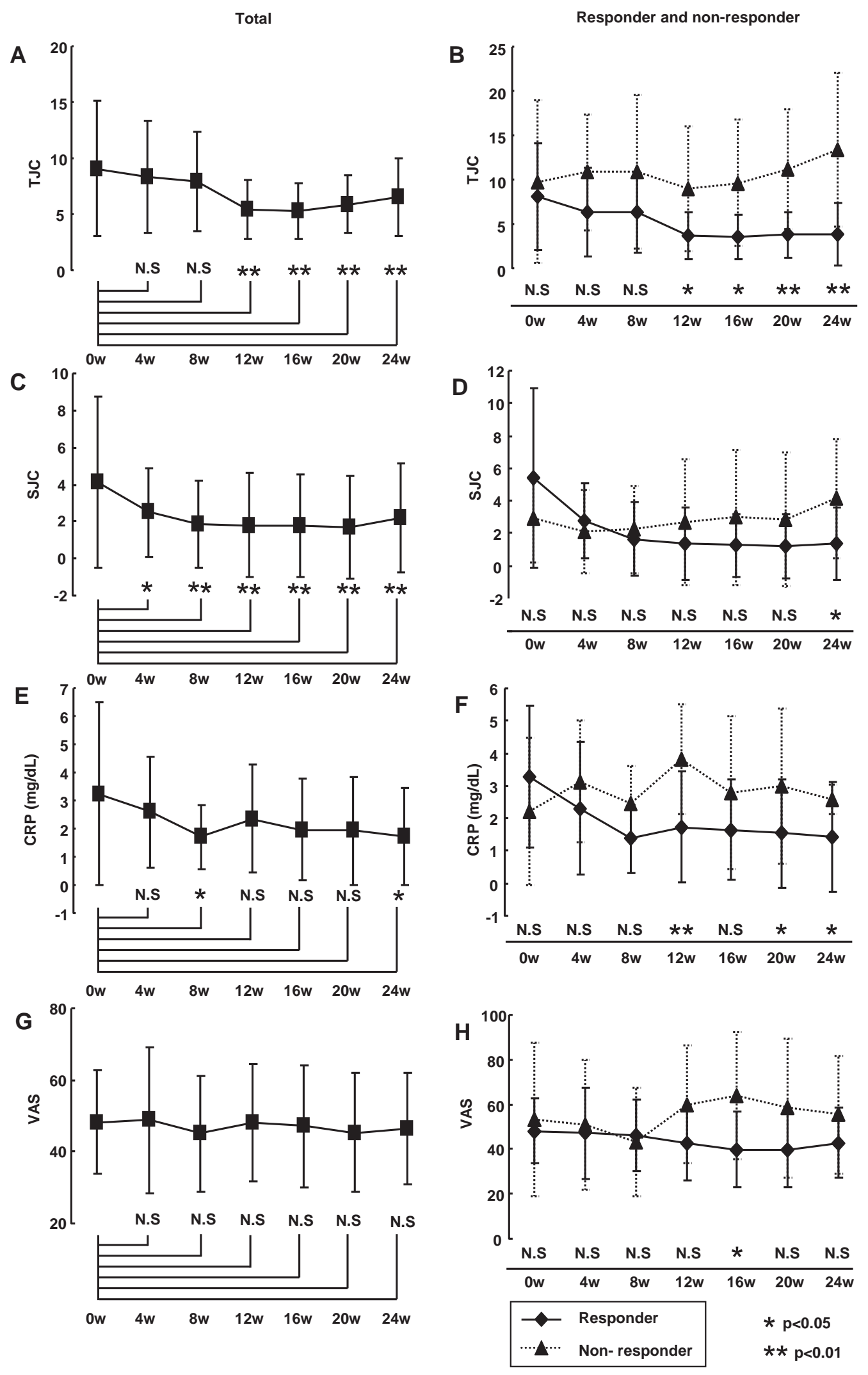

Figure 3. The efficacy value of TJC, SJC, CRP and VAS for 24 weeks. (A, C, E and G) Total, (B, D, F and H) Responder and non-responder. The responder group includes those patients who experienced a greater than or equal to moderate response after 24 weeks mizoribine (MZR) treatment. The non-responder group includes the remaining patients. TJC: tender joint counts, SJC: swollen Joint counts, CRP: C-reactive protein, VAS: visual analogue scale. N.S: not significant, ${ }^{*} \mathbf{p}<0.05$, ${ }^{* *} \mathbf{p}<\mathbf{0 . 0 1}, \mathbf{0}$ week vs each week in $\mathbf{A}, \mathbf{C}, \mathbf{E}$ and $\mathbf{G}$, responder vs non-responder in $\mathbf{B}, \mathrm{D}, \mathbf{F}$ and $\mathbf{H}$. 
Table 3. Clinical Evaluation of Responder and Non-Responder Groups. The Responder Group Includes Those Patients who Experienced a Greater Than Moderate Response after 24 Weeks MZR Treatment. The Non-Responder Group Includes the Remaining Patients. PSL: predonisolone, DMARDs: disease modifying anti-rheumatic drugs, TJC: Tenderness joint counts, SJC: Swollen joint counts, CRP: C-reactive protein, VAS: visual analogue scale, DAS28-CRP: the 28 joint count Disease Activity Score using $\mathrm{C}$ reactive protein

\begin{tabular}{l|c|c|c}
\hline & Responder & Non-responder & p-value \\
\hline Number (M:F) & $18(2: 16)$ & $14(4: 10)$ & 0.421 \\
Age (years) & $62.4 \pm 11.4$ & $66.5 \pm 8.60$ & 0.316 \\
Duration (years) & $14.1 \pm 10.2$ & $12.5 \pm 12.4$ & 0.702 \\
PSL (mg/day) & $4.35 \pm 3.09$ & $4.00 \pm 3.78$ & 0.789 \\
DMARDs & $0.88 \pm 1.11$ & $0.18 \pm 0.41$ & 0.057 \\
TJC & $8.06 \pm 6.02$ & $9.73 \pm 9.20$ & 0.565 \\
SJC & $5.41 \pm 5.55$ & $2.91 \pm 2.70$ & 0.178 \\
CRP $(\mathrm{mg} / \mathrm{dL})$ & $3.27 \pm 2.19$ & $2.22 \pm 2.27$ & 0.231 \\
VAS $(\mathrm{mm})$ & $45.9 \pm 15.1$ & $53.5 \pm 29.8$ & 0.375 \\
DAS28-CRP & $4.78 \pm 0.91$ & $4.73 \pm 1.49$ & 0.897 \\
BUN (mg/dL) & $17.7 \pm 3.22$ & $14.0 \pm 3.16$ & 0.192 \\
Cr (mg/dL) & $0.81 \pm 0.25$ & $0.55 \pm 0.18$ & 0.167 \\
Plasma level of MZR $(\mu \mathrm{g} / \mathrm{mL})$ & $2.20 \pm 0.49$ & $1.59 \pm 0.82$ & $0.020^{*}$ \\
\hline
\end{tabular}

Table 4. Analysis of Mizoribine Concentration and Clinical Efficacy

\begin{tabular}{c|cccc}
$\begin{array}{c}\text { Cut off } \\
(\mu \mathrm{g} / \mathrm{mL})\end{array}$ & PPV & NPV & Sensitivity & Specificity \\
\hline$\geq 2.73$ & 50 & 42.3 & 6.3 & 91.7 \\
$\geq 2.59$ & 66.7 & 43.5 & 23.5 & 83.3 \\
$\geq 2.42$ & 77.8 & 50.0 & 41.2 & 83.3 \\
$\geq 2.34$ & 81.8 & 55.6 & 52.9 & 83.3 \\
$\geq 2.21$ & 84.6 & 62.5 & 64.7 & 83.3 \\
$\geq 2.12$ & 81.3 & 69.2 & 76.5 & 75.0 \\
$\geq 2.07$ & 76.6 & 66.7 & 76.5 & 66.7 \\
$\geq 1.90$ & 72.2 & 63.6 & 76.5 & 58.3 \\
$\geq 1.88$ & 68.4 & 60.0 & 76.5 & 50.0 \\
$\geq 1.59$ & 71.4 & 75.0 & 88.2 & 50.0 \\
$\geq 1.3$ & 66.7 & 80.0 & 94.1 & 33.3
\end{tabular}

PPV; positive predictive value, NPV; negative predictive value

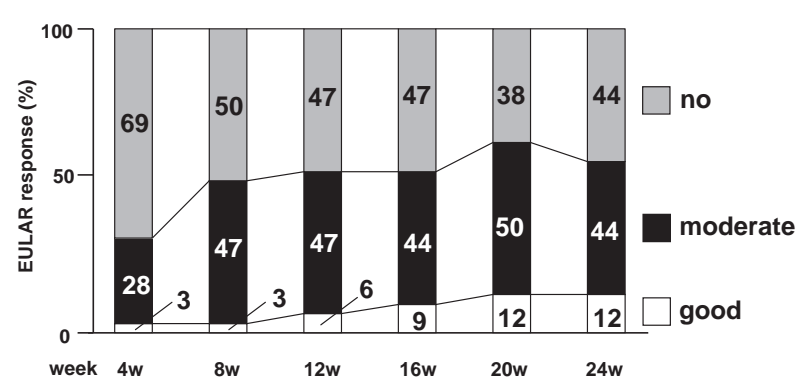

Figure 4. Clinical responses for arthrits are defined as improvement of the disease activity score (DAS) by the European League Against Rheumatism (EULAR) criteria. The number of patients at each time point is shown in the bar.

Given that the peak plasma level correlates with the clinical effect, we may consider up to $3-4 \mu \mathrm{g} / \mathrm{mL}$ to achieve a good response.

In contrast, there has been a report that bone marrow suppression, hepatic dysfunction, and severe stomatitis appeared when the trough level of MZR was $4 \mu \mathrm{g} / \mathrm{mL}$ or above in kidney transplantation recipients with marked renal dysfunction (14). Since MZR is excreted via the kidney, monitoring of the trough level is necessary to ensure safety in such patients. Accordingly, the dose of MZR should be carefully considered especially for elderly patients whose renal function assumed to be decreased with age.

Approximately ten types of oral DMARDs are currently available in Japan, and methotrexate (MTX) is now being prescribed as first-line therapy in many cases of RA. However, some of the patients cannot be given MTX treatment, because of RA associated lung disease, interstitial pneumonia, renal dysfunction or other complications. Although, 


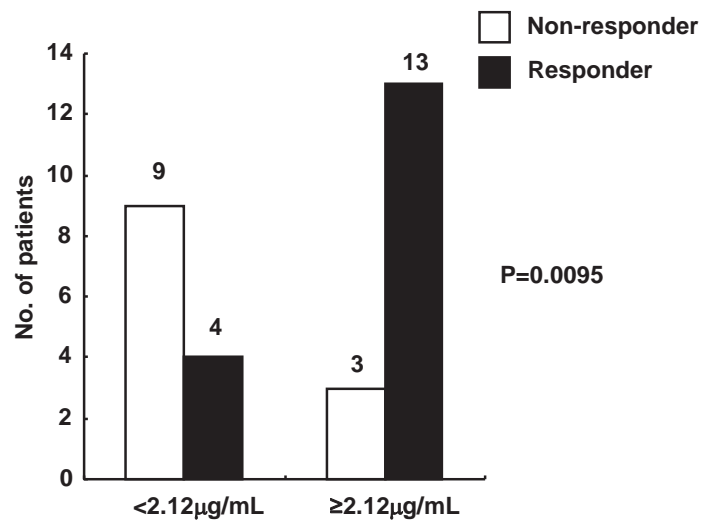

Figure 5. The relationship between the peak plasma level of mizoribine (MZR) and the clinical efficacy ( $p=0.0095)$.

MZR is considered a second-line DMARD (level of evidence B) in the guideline (29), we suggest that it can be often used in combination with other DMARDs, based on its mechanism of action and safety, especially in the elderly and patients with complications.

In the general use (three divided doses) of MZR, the correlation between the clinical effect and the peak plasma level of MZR has not been fully investigated in RA patients. We administrated MZR to 32 RA patients and monitored the relationship of its peak level and clinical efficacy. Our study included advanced RA patients who were not able to be treated with other general immunosuppressants and biologic agents because of complications and high age. In spite of this background, $56.3 \%$ (18/32 patients) of our cases could achieve a curative effect of greater than or equal to moderate response. Unfortunately, some of the patients did not have a clinical response even though they reached a high plasma level of MZR. We understand the clinical effect can not be explained by plasma level only, and it remains a matter to be discussed what other indicators might be involved.

In conclusion, this study indicated that optimal plasma level of MZR is important for the treatment of RA, especially in the elderly and patients with lung disease, renal dysfunction and/or other complications, and further evaluation is needed for the use of MZR therapy in long-term follow-up studies.

\section{Acknowledgement}

We wish to thank Asahi-Kasei Company, Tokyo, for the measurement of the plasma level of MZR and Jana Mooster for reading the manuscript.

\section{References}

1. Mizuno K, Tsujino M, Takada M, Hayashi M, Atsumi K. Studies on bredinin. I. Isolation, characterization and biological properties. J Antibiot (Tokyo) 27: 775-782, 1974.

2. Koyama H, Tsuji M. Genetic and biochemical studies on the activation and cytotoxic mechanism of bredinin, a potent inhibitor of purine biosynthesis in mammalian cells. Biochem Pharmacol 32:
3547-3553, 1983.

3. Kusumi T, Tsuda M, Katsunuma T, Yamamura M. Dual inhibitory effect of bredinin. Cell Biochem Funct 7: 201-204, 1989.

4. Hughes SE, Gruber SA. New immunosuppressive drugs in organ transplantation. J Clin Pharmacol 36: 1081-1092, 1996.

5. Inou T, Kusaba R, Takahashi I, et al. Clinical trial of bredinin in renal transplantation. Transplant Proc 13: 315-318, 1981.

6. Takei S. Mizoribine in the treatment of rheumatoid arthritis and juvenile idiopathic arthritis. Pediatr Int 44: 205-209, 2002.

7. Aihara Y, Miyamae T, Ito S, et al. Mizoribine as an effective combined maintenance therapy with prednisolone in child-onset systemic lupus erythematosus. Pediatr Int 44: 199-204, 2002.

8. Yoshioka K, Ohashi Y, Sakai T, et al. A multicenter trial of mizoribine compared with placebo in children with frequently relapsing nephrotic syndrome. Kidney Int 58: 317-324, 2000.

9. Kaneko K, Nagaoka R, Ohtomo Y, Yamashiro Y. Mizoribine for childhood IgA nephropathy. Nephron 83: 376-377, 1999.

10. Kasama T, Wakabayashi K, Odai T, et al. Effects of low-dose mizoribine pulse therapy in combination with methotrexate in rheumatoid arthritis patients with an insufficient response to methotrexate. Mod Rheumatol 19: 395-400, 2009.

11. Horikoshi $M$, Ito $S$, Ishikawa $M$, et al. Efficacy of mizoribine pulse therapy in patients with rheumatoid arthritis who show a reduced or insufficient response to infliximab. Mod Rheumatol 19: 229-234, 2009.

12. Sakai K, Matsuno H, Tsuji H, Sainou H, Yashima S. Synocial fluid and plasma levels of mizoribine in RA patients. Jpn J Inflamm 14: 521-524, 1994 (in Japanese).

13. Tanaka H, Suzuki K, Nakahata T, Tsugawa K, Ito E, Waga S. Mizoribine oral pulse therapy for patients with disease flare of lupus nephritis. Clin Nephrol 60: 390-394, 2003.

14. Sonda K, Takahashi K, Tanabe $K$, et al. Clinical pharmacokinetic study of mizoribine in renal transplantation patients. Transplant Proc 28: 3643-3648, 1996.

15. Kawasaki Y, Hosoya M, Kobayashi S, et al. Oral mizoribine pulse therapy for patients with steroid-resistant and frequently relapsing steroid-dependent nephrotic syndrome. Nephrol Dial Transplant 20: 2243-2247, 2005.

16. Nishioka Y, Horita Y, Tadokoro M, et al. Changing mizoribine administration from three divided doses to one single dose induced remission of relapsed membranous nephropathy. Nephrol Dial Transplant 21: 2337-2338, 2006.

17. Steinbrocker O, Traeger $\mathrm{CH}$, Batterman RC. Therapeutic criteria in rheumatoid arthritis. J Am Med Assoc 140: 659-662, 1949.

18. Nakamura N, Nishimura K, Nishino J, Fukunaga S, Kochi A, Matsushita T. Single dose mizoribine at 100 or $50 \mathrm{mg} /$ day for rheumatoid arthritis: efficacy and safety profile results of 6-month administration. Mod Rheumatol 17: S112, 2007.

19. Nishimura K. Prevention of joint destruction in rheumatoid arthritis patients receiving combination mizoribine and methotrexate therapy. Pharm Medica 26: 134-135, 2008 (in Japanese).

20. Kuroda T, Hirose S, Tanabe N, et al. Mizoribine therapy for patients with lupus nephritis: the association between peak mizoribine concentration and clinical efficacy. Mod Rheumatol 17: 206212, 2007.

21. Tokuda M, Dobashi H, Hiraishi T, Mitsunaka H, Takahara J, Hirohata M. Effect of mizoribine pulse therapy on the disease activity of rheumatoid arthritis refractory to the treatment with methotrexate. Rheumatology 20: 519-526, 1998 (in Japanese).

22. Kohriyama K, Hiramatsu Y, Aoyama T. Efficacy of combination pulse therapy with methotrexate and mizoribine for patients with rheumatoid arthritis showing escape phenomenon to low-dose methotrexate therapy. Rinsho Riumachi 15: 227-234, 2003 (in Japanese).

23. Murai T, Arai K, Fujisawa J, Kondo N, Hanyu T. Evaluation of combination pulse therapy with methotrexate and mizoribine for 
patients with methotrexate-resistant rheumatoid arthritis. J Chubu Rheum Assoc 37: 80-81, 2006 (in Japanese).

24. Shida J, Shuto T, Tokito T, et al. Clinical investigation of efficacy by administration of mizoribine once a day for rheumatoid arthritis. Kyushu Riumachi 26: 9-14, 2006 (in Japanese).

25. Tanaka H, Mine T, Tani $\mathrm{Y}$, Chagawa K, Taguchi T. Combination therapy with methotrexate and mizoribine for patients with rheumatoid arthritis. Orthop Traumatol 57: 439-442, 2008 (in Japanese).

26. Takada K, Asada S, Ichikawa Y, et al. Pharmacokinetics of bredinin in renal transplant patients. Eur J Clin Pharmacol 24: 457 $461,1983$.
27. Katayama A, Uchida K, Goto N, et al. Evaluation of the blood mizoribine concentration during high-dose mizoribine administration $(10 \mathrm{mg} / \mathrm{kg} / \mathrm{day})$ in the early period after kidney transplantation (Part 2). Ther Res 24: 986-988, 2003 (in Japanese).

28. Tsubouchi Y, Fukuda W, Kawahito Y, et al. A case of lupus nephritis improved after appropriately adjusting the dosage of mizoribine. Mod Rheumatol 18: 91-95, 2008.

29. Study Group of the Ministry of Hearth, Labour and Welfare. Diagnostic and therapeutic manual of rheumatoid arthritis. Diagnostic manual and evidence-based treatment guidelines. Tokyo: Japan Rheumatism Foundation 2004 (in Japanese).

(C) 2010 The Japanese Society of Internal Medicine http://www.naika.or.jp/imindex.html 